Case of the Month

\title{
Aleukemic bcr-abl positive granulocytic sarcoma
}

\author{
Jew-Win Kuan ${ }^{\mathrm{a}, *}$, Rajadurai Pathmanathan ${ }^{\mathrm{b}}$, Kian-Meng Chang ${ }^{\mathrm{a}}$, Sen-Moi Tan ${ }^{\mathrm{a}}$ \\ a Hematology Department, Ampang Hospital, Jalan Mewah Utara, Pandan Mewah, 68000 Ampang, Selangor, Malaysia \\ b Pathology Department, Sime Darby Medical Centre Subang Jaya, 1, Jalan SS 12/1A, 47500 Subang Jaya, Selangor, Malaysia
}

\section{A R T I C L E I N F O}

\section{Article history:}

Received 27 October 2008

Received in revised form 13 January 2009

Accepted 15 January 2009

Available online 12 February 2009

\section{Keywords:}

Granulocytic sarcoma

Bcr-abl

CML

\begin{abstract}
A B S T R A C T
Granulocytic sarcoma (GS) can occur de novo or in association with intramedullary myeloid disorders. With the advent of sophisticated molecular detection techniques to detect diagnostic genes such as bcr$a b l$, PML-RARA and CBFB/MYH11 in bone marrow or peripheral blood, many cases of the so called 'primary' GS are questionable. We report a case of primary GS where the tumor mass bcr-abl translocation was demonstrated by fluorescent in situ hybridization in which there was no evidence of chronic myeloid leukemia (CML). This is an important finding as it highlights the possibility that CML may present as a sole extramedullary form, and illustrates potential treatment by tyrosine kinase inhibitor.
\end{abstract}

(c) 2009 Elsevier Ltd. All rights reserved.

\section{Introduction}

A myeloid sarcoma (MS) is a tumor mass consisting of myeloid blasts with or without maturation occurring at an anatomical site other than the bone marrow [1]. Infiltrations of any site of the body by myeloid blasts in leukaemic patients are not classified as MS unless they present with tumor masses in which the tissue architecture is effaced [1]. This condition was first described by Burns in 1811 [2]. King used 'chloroma' as the first term to describe this condition in 1853 [3]. The World Health Organization (WHO) has renamed the condition as MS and divided it into two major categories, granulocytic sarcoma (GS) and monoblastic sarcoma [4]. GS can arise de novo or can present concurrently with, after, or upon relapse with the diagnosis of myeloid disorders. The best-known concurrent myeloid disorder is acute myeloid leukemia (AML) [5]. Most untreated primary GS will progress into AML within 11 months [6,7]. Although the disease is associated with chronic myeloid leukemia (CML) [5], myeloproliferative disorders (MPD) [7,8] and myelodysplastic syndrome (MDS) $[9,10]$, these conditions were never truly preceded by primary GS. $B c r-a b l$ fusion product is classically due to chromosomal translocation $t(9 ; 22)(\mathrm{q} 34 ; \mathrm{q} 11)$ in CML. With the advent of fluorescent in situ hybridization (FISH), bcr-abl translocation can be demonstrated in the tumor cells of GS. As far as we know, this is the first reported case of FISH bcr-abl positive GS without CML and AML.

\footnotetext{
* Corresponding author. Tel.: +60 198593011; fax: +60 362501864.

E-mail address: kuanjewwin@hotmail.com (J.-W. Kuan).
}

\subsection{Case report}

A 21-year-old Malay student presented to an otorhinolaryngologist with bilateral bleeding nasal polyps in August 2006. Diagnosis of GS was made from the bilateral excisional biopsy. The biopsy showed immature lymphoid-type cells strongly positive for CD45 and negative for CD3, CD20, MNF116, S100, NSE, HMB-45, ALK-1, and CD30. The cells exhibit focal positivity for $\mathrm{CD} 43$, lysozyme and myeloperoxidase (MPO). The imaging evaluation revealed sinonasal polypoidal masses extending into the right maxillary, ethmoid, sphenoid and frontal sinuses. Bilateral nodal masses in jugulodigastric chain and posterior triangles of the neck were also seen. His full blood count (FBC), peripheral blood film (PBF) and bone marrow (BM) examination were normal. BM cytogenetic examination showed a normal karyotype. He underwent AML-like chemotherapy. He completed two courses of idarubin and cytarabine induction chemotherapy, and one course of high dose cytarabine as consolidation. Unfortunately, he was lost to follow up after that.

Seven months later in late July 2007 he represented with recurrent and more extensive nasal tumor involving the paranasal sinuses, left orbital cavity and anterior cranial fossa. The same histopathological and immunological findings as in the initial nasal biopsy were seen with strong CD43 positivity. FBC, PBF and $\mathrm{BM}$ examination were normal. BM immunophenotyping did not detect blast in the CD34/117 gate. BM cytogenetic was a short spread, but FISH bcr-abl (LSI bcr-abl dual colour, dual fusion probe Vysis, Abbott) was negative. He was given induction therapy with idarubicin, cytarabine and intrathecal triple agents. While waiting for local radiotherapy, he was given etoposide and cytarabine in September 2008, when at the same time a new scalp swelling at right vertex region was noted. Although computed 